\title{
Imaging contribution for the diagnosis of carotidynia
}

\author{
Antonio Jose da Rocha $\cdot$ Eduardo Hideki Tokura • \\ André Paciello Romualdo - Mathias Fatio · \\ Hugo Pereira Pinto Gama
}

Received: 30 November 2008/ Accepted: 14 January 2009/Published online: 7 February 2009

(C) Springer-Verlag 2009

\begin{abstract}
Carotidynia is a condition characterized by tenderness of the carotid artery and ipsilateral neck pain, eventually aggravated by head movements, swallowing, coughing and sneezing. We report a 52-year-old male presenting cervical pain associated with localized tenderness and reversible structural changes of the common carotid wall, emphasizing the applicability of the diagnostic imaging methods for the presumptive diagnosis of carotidynia in the appropriate clinical context.
\end{abstract}

Keywords Carotidynia - Neck pain · Facial neuralgia . Imaging methods

\section{Introduction}

The term carotidynia was first used in 1927 by Fay [1] to describe the tenderness over the carotid bifurcation, sometimes associated with "atypical facial neuralgy". Over the years it became to be known as a distinct entity, particularly when Roseman [2] in 1967, characterized it by two cardinal signs: tenderness of the carotid artery and ipsilateral neck pain.

\footnotetext{
A. J. da Rocha · H. P. P. Gama

Santa Casa de São Paulo, Fleury Medicina Diagnostica,

São Paulo, Brazil

E. H. Tokura - A. P. Romualdo · M. Fatio

Fleury Medicina Diagnostica, São Paulo, Brazil

H. P. P. Gama ( $\square)$

Rua Cincinato Braga 282, Bela Vista, São Paulo, SP 01333-910, Brazil

e-mail: hugo.gama@fleury.com.br
}

By meeting specific criteria, the International Headache Society (IHS) Classification Committee [3] recognized in 1988 acute idiopathic carotidynia as a specific entity. However, some authors questioned the evidence that support the concept of carotidynia [4] and the current definition of IHS classification [5] considers, since 2004, the existence of a syndrome including many varieties of pain in the carotid region.

In this article, we report a case presenting cervical pain associated with localized tenderness and reversible structural changes of the common carotid wall, with the intent to emphasize the applicability of the diagnostic imaging methods that support the diagnosis of carotidynia, in the appropriate clinical context.

\section{Case report}

A 52-year-old male presenting localized cervical pain in the anterior-right aspect of the neck, at the level of the carotid artery. Ultrasound showed a focal thickening of the common carotid artery, exactly at the place where the patient refers the presence of pain. Doppler (Fig. 1a) did not show any significant hemodynamic changes. The patient had no fever and only showed a discrete increase of the erythrocyte sedimentation rate (ESR) $14 \mathrm{~mm}$, and a relative increase of the C-Reactive Protein (CRP) value $0.08 \mathrm{mg} / \mathrm{dL}$. MRI and angio-MRI showed a focal wall thickening, isointense on T1 weighed-images (WI), more pronounced in the medial right common carotid wall, with impregnation by gadolinium, but no significant intraluminal stenosis (Fig. 1b, c).

Based on the clinical and imaging criteria, the diagnosis of carotidynia was made and a therapeutical approach with corticosteroids for 4 months, with a maximum dose of 
Fig. 1 Doppler ultrasound (a) shows focal thickening of the right common carotid artery (RCCA), without hemodynamic changes. MRI and angio-MRI (b and c) demonstrates focal wall thickening in the medial RCCA, with impregnation by gadolinium and minimal narrowing, but no significant luminal stenosis. Follow-up MRI (d) shows complete remission of the structural changes
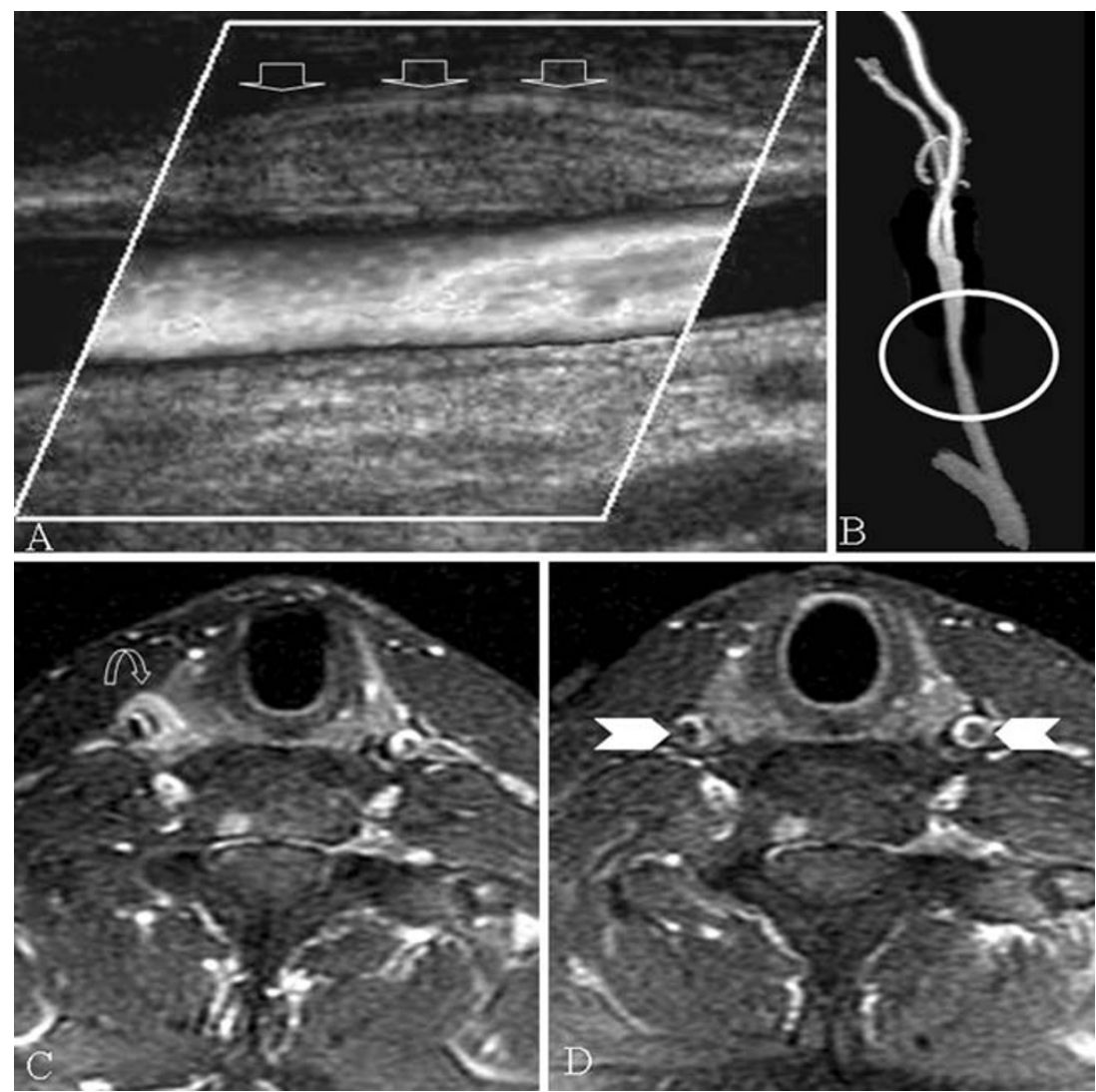

$80 \mathrm{mg} /$ day, was suggested. Follow-up showed a favorable clinical course, normal ESR and CRP values $(6 \mathrm{~mm}$ and $0,02 \mathrm{mg} / \mathrm{dL}$, respectively) and complete remission of the structural changes documented by imaging methods (Fig. 1d).

\section{Discussion}

The recognition of carotidynia as a specific entity has been the object of debate [3-5]. The main clinical findings described include cervical pain and localized tenderness that may last for a variable amount of time. However, the differential diagnosis list can be extensive and includes carotid diseases such as giant cell arteritis, thrombosis, fibromuscular dysplasia, aneurysm and dissection or nonvascular diseases, such as lymphadenitis, spinal degenerative processes and syaloadenitis.

To the best of our knowledge, there is no reported large series of cases correlating histologic and imaging features. We only found one case report showing thickening and swelling of the adventitia, presenting at the histological analysis a chronic inflammatory process, associated to vascular and fibroblasts proliferation and without any evidence of microorganisms or consistent signal of vasculitis [6]. The complete remission of the symptoms and structural changes in a few days after corticotherapy in the case reported here corroborates the possibility of inflammatory etiology.

Some recently published series of cases have defined some structural abnormalities, demonstrated by different imaging methods, which contribute to the study of carotidynia [7]. CT and digital angiography supply little relevant information, therefore having limitations for this specific setting.

Our imaging findings are in line with those previously described. We mainly emphasize the focal eccentric thickening of the carotid wall at the level of the pain referred by the patient, without any hemodynamic changes at the Doppler study or changes of the vascular lumen at angio-MRI. Despite some articles suggesting the involvement of two layers of the vessel's wall, we believe that structural changes are based mainly on the adventitia, thus explaining the eccentric growth of the lesion. The MRI study with gadolinium allows a more detailed analysis of the lesion and shows its relation to the vessel wall. Furthermore, MRI allows the depiction of other changes of the vessel wall, as well as other differential diagnoses, particularly arterial dissection, which was excluded because the absence of intramural hematoma (hyperintense crescent within vessel wall on fat-saturated T1-WI) and intimal flap. 
Facing a case of cervical pain, the exclusion of other factors is mandatory. However, we consider that the demonstration of a focal and eccentric structural lesion presumably located at the adventitia layer of the carotid artery and in close correlation with the tenderness and painful point, in addition to a good response to corticotherapy, is the most appropriate approach to make the presumptive diagnosis of carotidynia.

The diagnostic imaging methods, particularly MRI, constitute a useful complementary tool for the diagnosis and choice of appropriate treatment of suspected cases. The diagnostic parameters should be gradually clarified and we believe that MRI can bring relevant information to this discussion and better use of the term carotidynia.

Conflict of interest None.

\section{References}

1. Fay T (1927) Atypical neuralgia. Arch Neurol Psychiatry 18:309315

2. Roseman DM (1967) Carotidynia. Arch Otolaryngol 85:81-84

3. Headache Classification Committee of the International Headache Society (1988) Classification and diagnosis criteria for headache disorders, cranial neuralgias and facial pain. Cephalalgia 8(Supp17): $48-49$

4. Biousse V, Bousser MG (1994) The myth of carotidynia. Neurology 44:993-995

5. Headache Classification Subcommittee of the International Headache Society (2004) The international classification of headache disorders, 2nd edn. Cephalalgia 24(Suppl 1):9-160

6. Upton PD, Smith JG, Charnock DR (2003) Histologic confirmation of carotidynia. Otolaryngol Head Neck Surg 129:443-444

7. Kosaka N, Sagoh T, Uematsu H, Kimura H, Miyayama S, Noguchi M, Itoh H (2007) Imaging by multiple modalities of patients with a carotidynia syndrome. Eur Radiol 17:2430-2433 